\title{
Improvement and Innovation of Appearance Design of Coffee Machine
}

\author{
Zhao Zhanfeng \\ Wenzhou Vocational \& Technical College, Wenzhou, 325035 \\ Zhanfeng@qq.com
}

Keywords: Coffee machine, Innovation, Design

\begin{abstract}
In China, traditional manufacturing industries are changing into innovative industries, and the labels of commodities are changing from "made in China" to "created in China. The design and production technology of the coffee machines have started early abroad, which are relatively mature, focus on innovative design and development. However, market positioning of most domestic coffee machines is not clear without innovation, just the introduction of foreign design and technology. It would cost much to model or copy domestic and foreign existing products because it would be costly to process with heavy dependence on others. Modeling functions are mostly stereotyped, which are "stiff" and lack a sense of design, and there are many shortcomings in the using functions. So there is a big space for improvement and development.
\end{abstract}

\section{Classification of coffee machines}

\subsection{Semi-automatic coffee machine}

Maintenance of semi-automatic coffee machine is an easy, reliable work. The coffee made by semi-automatic coffee machine tastes silky. But the drawback is that the operation is cumbersome and complex, the operator must undergo strict training to use this machine to make high-quality coffee and improve the work efficiency.

\subsection{Automatic coffee machine}

Automatic coffee machine boasts convenient and fast operation, high efficiency and consistent quality. The number of cup of coffee per hour made by it is 60 to 280 , which is quite more than the traditional coffee machine, so it is preferred by the majority of consumers. But its structure is more complex, requires expensive maintenance. The main manufacturers of automatic coffee machine are Germany, Switzerland and Italy. Among them, Swiss automatic coffee machine design is ingenious and unique. Automatic coffee machines made in Italy are simple in appearance, stable and reliable in quality, enjoying high reputation in the international coffee machine industry. They can be subdivided into three sizes: large, medium and small, which are suitable for office usage and family usage, as shown in figure 1 . 


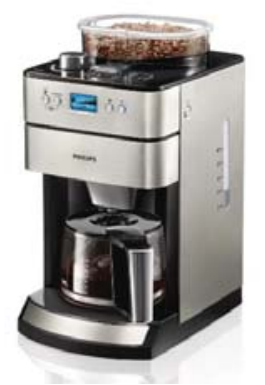

Fig. 1 Automatic coffee machine

\subsection{Survey of coffee machine appearance}

There are many kinds of coffee machines. The models of coffee machines sold in the market are various, from simple geometry to full arcs and strange shapes. The appearance shapes and the styles are different. But from the point of the whole design, the appearance of coffee machine modeling is square, with simplicity as the mainstream, and the overall shape is relatively clumsy and lacks of aesthetic design.

\section{Human-computer dimension analysis of coffee machine}

Taking the normal area associated with the hands action into account, the man-machine size diagram is shown in figure 2 .

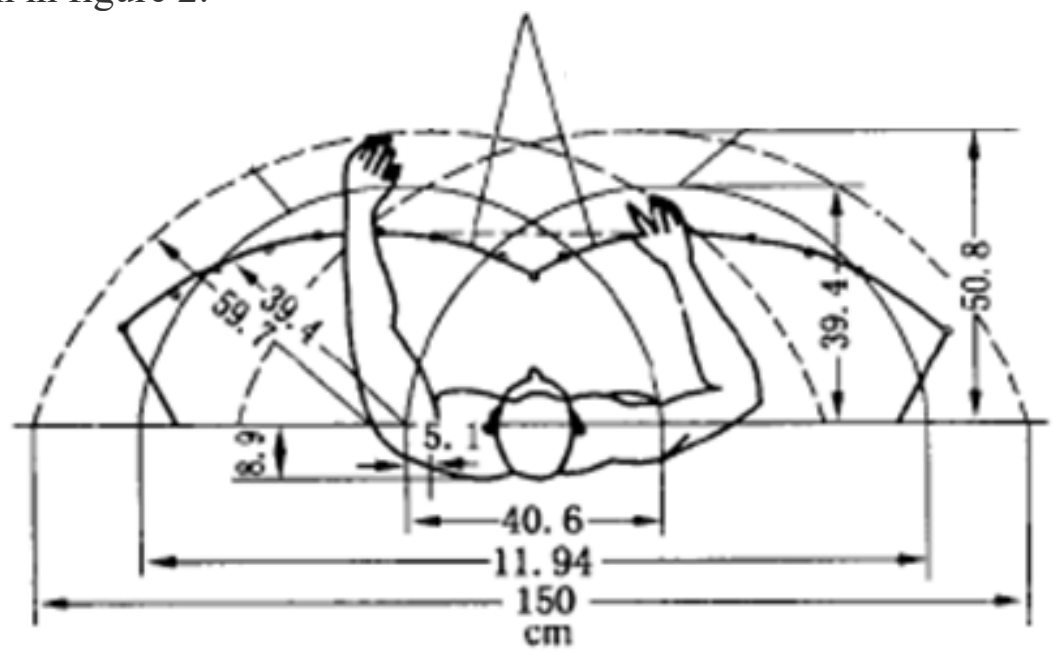

Fig. 2 man-machine dimensions

\subsection{Analysis of advantages and disadvantages of coffee machine}

First of all, the advantages of the coffee machine on the market are that they have a variety of appearances, which are easy to understand, easy to operate, and time-and-effort saving. Users who have no experience in coffee making can also easily produce delicious coffee, bringing much convenience in practice. Secondly, the coffee machine can produce different tastes and different styles of coffee according to the requirements of users, and better meet the diversified needs of family members. 


\section{Preliminary design scheme}

\subsection{Specific positioning of design}

Specific positioning for appearance design of home automatic coffee machine

In terms of product modeling, the domestic coffee machine is a highly functional product, whose shape is greatly influenced by the functionality of the product itself. It not only makes the appearance of the product conform to the rule of normal aesthetics but also coordinates the relationship among function, performance and structure of the product. Through the investigation, the author has found that the coffee machines in the domestic market are mostly cuboid, the overall shape is rather clumsy lacking in a sense of beauty. Thus, it is necessary to change the shape of the coffee machines in the past and adopt streamlined design to minimize the volume and weight of the coffee machines, making machines embody the miniaturization and endowing the coffee machine product with semantics. And meanwhile, the coffee machine appearance should be succinctly generous without losing the sense of fashion.

In terms of functions, most coffee machines on the market are mainly used for grinding, filling, pressing, brewing, extracting, and removing residue. However, there are still some deficiencies, such as cumbersome process and inconvenient operation. So the author has improved the design in these aspects, in addition, the author added some functions according to the needs of customers, such as intelligent touch screen, timing heat preservation, music warning function, automatic energy-saving standby function.

As for structure, it is known that the structure of the coffee machine has crusher, water tank, heater, brewer, filter, waste recycling box, etc. The key to the design of the automatic coffee machine lies in the heater, brewer and control system. During the process of designing, the structural space is necessary to ensure its cubage.

\subsection{Hand-drawn sketches}

Some indispensable structures of coffee machine, under the premise of reasonable design, have been changed with other functions. Several hand-drawn sketches are shown as Figure 3, 4.

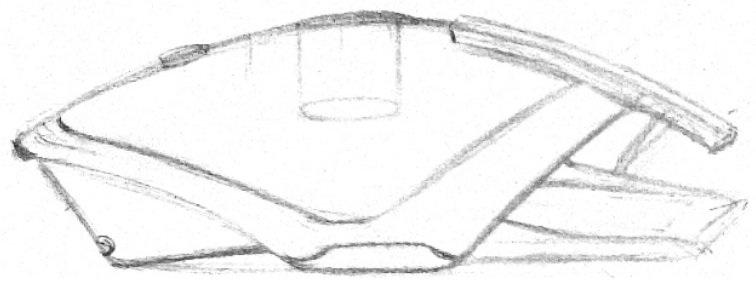

Figure 3 Sketch 1

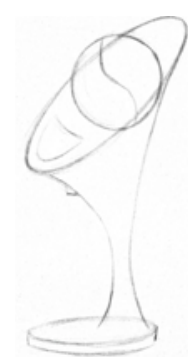

Figure 4 Sketch 2

This design is inspired by the modeling of mouse. The key point of the design is drawing 
structure of the dripping nozzle and the hydroponic plate, which can be pulled out when it needs to be used, and automatically retracted when it is not used. The chassis can be placed on top of the coffee cup when it is not used, and the chassis retracts into the body to show the function of storage.

This design uses the simple image of lily, which is simple and generous. The ball is a bean container and a water tank that can be rotated.

\subsection{Selection of design options}

The selection of the design scheme is to select the best design by comparing and analyzing the previous sketches. After drawing some sketches, a better design sketch is selected and a product is processed according to this sketch. This process of selection is based on the semantics of the coffee machine, the creative appearance design, as well as the rationality of the overall modeling structure. And the sketches are shown in figure 5, 6 as below.

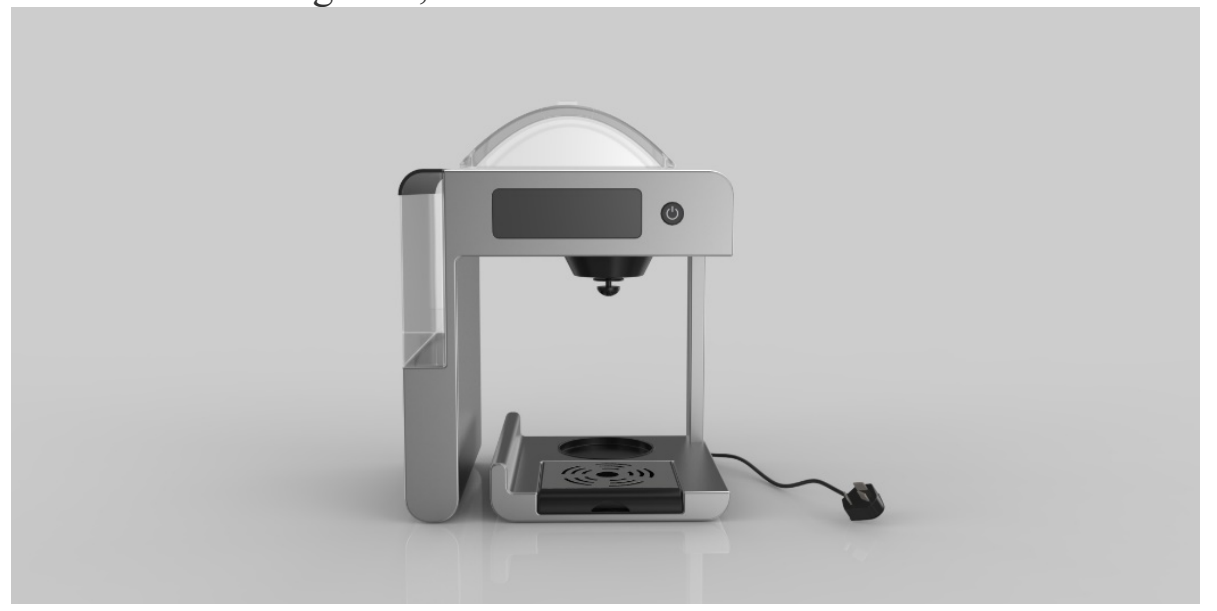

Figure 5 Rendering

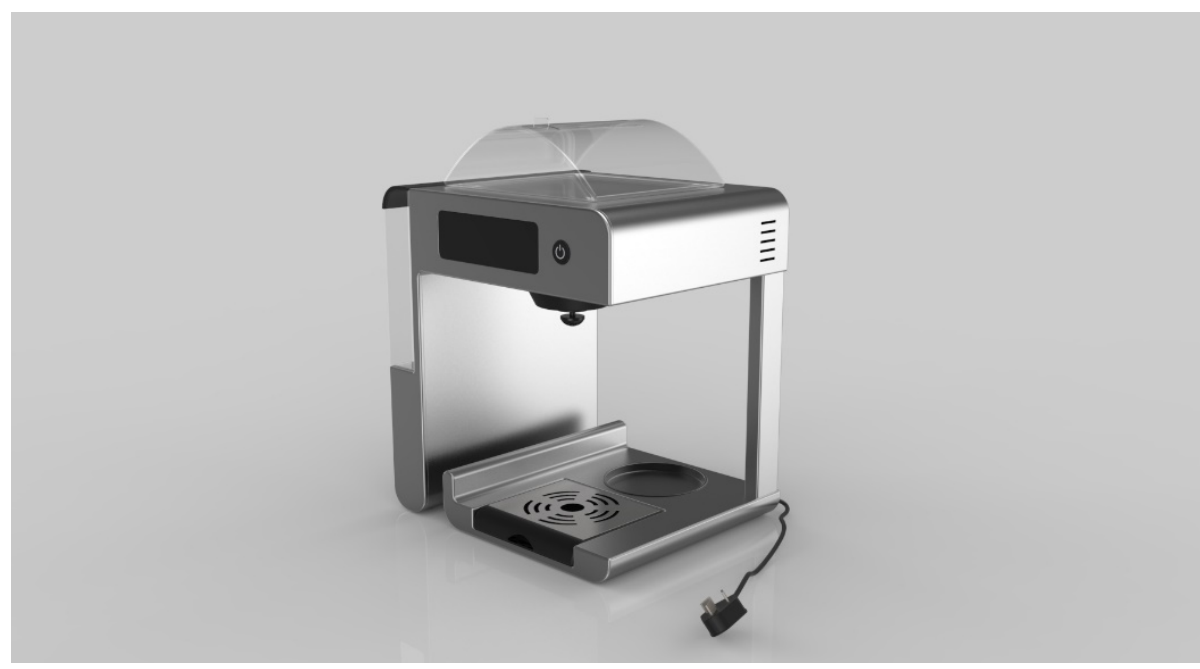

Figure 6 Details

\section{Conclusion}

This innovative product is a breakthrough against the past modeling design. It has improved and innovated on the basis of the existing coffee machine, so the product modelling is simple with its highlights. It pays attention to the function of the product, and has improved the shortcomings of 
the existing coffee machine. It pursues multi-functional integration, making the product operation easy and safe. And it also pays attention to the functions that are approachable to newbies and the commonality of the products. Through the research and investigation of the various parts of the products, his machine can be made to better conform to the ergonomics of the products, maximize the comfortableness of the consumers, and enable the consumers operate them healthily and safely. And this machine can save resources as much as possible. In addition, the durability of the product is guaranteed. This product has improved the appearance as well as the life cycle. With the combination of modern science and technology, the product has a sense of high-fashion, reflecting the trend of science and technology at present or even in the future.

\section{References}

[1] Li Danbilin. Discussion on Product Redesign Based on Sustainable Idea[J]. Packaging Engineering, 2007, 28 (1):168.

[2] Chinese Commerce Network. Research Report on the Deep Investigation on the Market of the Coffee Machine and Its Developmental Trends from 2011 to 2015[EB/OL]. (2010-12-20) [2014-02-17]. http://kybg.askci.com/2010-11/20101125154522.html.

[3] Luo Shijian. User Experience and Product Innovation Design[M]. Beijing: Mechanical Industry Press,2010.

[4] Liu Baoshun. Structural Design of Product[M]. Beijing: China Building Industry Press,2009.

[5] Liu Yongxiang, Zhang Yao. Study on Universal Packing Design and Its Environmental Utility Based on the Theory of Make the Best Use of Everything [J]. Packaging Engineering, 2010,31(8):1—3. 